\title{
TEOR DE NITRATO COMO INDICADOR COMPLEMENTAR DA DISPONIBILIDADE DE NITROGÊNIO NO SOLO PARA O MILHO(1)
}

\author{
Lisandro Rambo ${ }^{(2)}$, Paulo Regis Ferreira da Silva ${ }^{(3)}$, Cimélio Bayer ${ }^{(3)}$, \\ Gilber Argenta $^{(4)}$, Mércio Luiz Strieder ${ }^{(5)}$, \& Adriano Alves da Silva ${ }^{(5)}$
}

\section{RESUMO}

Avanços na adubação nitrogenada em cobertura em milho poderão ser obtidos com a inclusão de características de solo como indicadores complementares da disponibilidade de N. Os objetivos deste estudo foram avaliar o potencial de uso, o nível crítico e o melhor estádio de desenvolvimento da cultura para determinação do teor de $\mathrm{N}_{-} \mathrm{NO}_{3}{ }^{-}$no solo, visando à predição da disponibilidade de $\mathrm{N}$ ao milho, e verificar se a determinação do teor de $\mathrm{N}-\mathrm{NH}_{4}{ }^{+}$, em adição ao teor de $\mathrm{N}-\mathrm{NO}_{3}{ }^{-}$, aumenta a eficiência na avaliação da disponibilidade de N. Para isso, realizou-se um experimento por dois anos agrícolas (2002/03 e 2003/04) em Argissolo Vermelho da Depressão Central do RS, no qual se realizou a simulação de diferentes níveis de disponibilidade de $\mathrm{N}$ no solo a partir da utilização de cinco doses de $N(0,50,100$, 200 e $\left.300 \mathrm{~kg} \mathrm{ha}^{-1}\right)$, parte na semeadura $(20 \%)$ e o restante em cobertura (estádio V3). O experimento seguiu o delineamento de blocos casualizados, com quatro repetições, e nos dois anos agrícolas foram avaliados os teores de $\mathrm{N}_{-} \mathrm{NO}_{3}{ }^{-}$, de $\mathrm{N}-\mathrm{NH}_{4}{ }^{+}$e de $\mathrm{N}$ mineral $\left(\mathrm{N}-\mathrm{NO}_{3}{ }^{-}+\mathrm{N}_{-} \mathrm{NH}_{4}{ }^{+}\right)$no solo $(0-30 \mathrm{~cm})$, em diferentes estádios de desenvolvimento (V3, V6, V10 e espigamento), e o rendimento de grãos do milho. Em geral, os teores de $\mathrm{N}_{-} \mathrm{NO}_{3}{ }^{-}$no solo foram sensiveis às doses de $\mathrm{N}$ aplicadas, com destaque para o estádio V6, no qual se verificou também a melhor relação desse elemento com o rendimento de grãos do milho. O nível crítico de $\mathrm{N}_{-} \mathrm{NO}_{3}{ }^{-}$no solo, a partir do qual a resposta à aplicação de $\mathrm{N}$ é improvável, foi estimado em $20 \mathrm{mg} \mathrm{kg} \mathbf{~ k g}^{-1}$ para o solo estudado. A avaliação do teor de $\mathrm{N}-\mathrm{NH}_{4}{ }^{+}$do solo, em adição ao teor de

(1) Extraído da tese de doutorado do primeiro autor. Pesquisa financiada pelo CNPq. Recebido para publicação em fevereiro de 2006 e aprovado em fevereiro de 2007.

(2) Engenheiro-Agrônomo, Dr., Desenvolvimento de Produtos, Syngenta Seeds Ltda. Rod. BR 452, km $142+543$ m. Caixa Postal 585, CEP 38400-974 Uberlândia (MG). E-mail: lisandrorambo@yahoo.com.br

(3) Professor da Faculdade de Agronomia, Universidade Federal do Rio Grande do Sul - FA/UFRGS. Av. Bento Gonçalves 7712 , CEP 91540-000 Porto Alegre (RS). Pesquisador do CNPq. E-mails: paulo.silva@ufrgs.br; cimelio.bayer@ufrgs.br

(4) Engenheiro-Agrônomo, Dr., Gerente Desenvolvimento de Produtos - Syngenta Seeds Ltda. E-mail: gilber.argenta@syngenta.com

(5) Engenheiro-Agrônomo, Aluno do Programa de Pós-graduação em Fitotecnica da FA/UFRGS. Bolsista do CNPq. E-mails: domercio@gmail.com; agroadriano@terra.com.br 
$\mathrm{N}-\mathrm{NO}_{3}{ }_{3}^{-}$, melhorou a predição da disponibilidade de $\mathrm{N}$ do solo, como evidenciado pela maior relação deste indicador com o rendimento de grãos, destacando a necessidade de desenvolvimento de kits de determinação rápida do teor de $\mathrm{N}$ mineral $\left(\mathrm{N}-\mathrm{NO}_{3}{ }^{-}+\mathrm{N}-\mathrm{NH}_{4}{ }^{+}\right)$no solo. Os resultados do uso de $\mathrm{N}-\mathrm{NO}_{3}{ }^{-}$e $\mathrm{N}-\mathrm{NH}_{4}{ }^{+}$como indicadores complementares da disponibilidade de $\mathrm{N}$ do solo para o milho são promissores, e estudos deverão ser desenvolvidos em diferentes condições edafoclimáticas para confirmar a adequação de seu uso no manejo da adubação nitrogenada em cobertura no milho.

Termos de indexação: Zea mays, eficiência de uso do $\mathrm{N}$, testes de nitrato, rendimento de grãos, estádios de desenvolvimento da planta.

\title{
SUMMARY: NITRATE CONCENTRATION AS A COMPLEMENTARY INDICATOR OF SOIL NITROGENAVAILABILITY TO CORN
}

\begin{abstract}
Nitrogen fertilization applied as sidedressing in corn can be optimized by considering additional soil characteristics as complementary indicators of soil $N$ availability. The objectives of this study were to evaluate the potential use, critical level and best sampling time of the soil nitrate $\left(\mathrm{NO}_{3}-\mathrm{N}\right)$ concentration to predict soil $\mathrm{N}$ availability in corn, as well as to verify if the determination of the soil ammonium $\left(\mathrm{NH}_{4}^{+}-\mathrm{N}\right)$ concentration, in addition to $\mathrm{NO}_{3}-\mathrm{N}$ concentration, enhances the efficiency of $\mathrm{N}$ availability evaluation. A field experiment was conducted in two growing seasons (2002/03 and 2003/04) in Eldorado do Sul, state of Rio Grande do Sul, Brazil. Different levels of soil N availability were simulated by the application of five $N$ rates $\left(0,50,100,200\right.$, and $\left.300 \mathrm{~kg} \mathrm{ha}^{-1}\right) 20 \%$ at sowing and the rest sidedressed at V3. The experiment was structured in a randomized block design with four replications. $\mathrm{NO}_{3}^{-}-\mathrm{N}, \mathrm{NH}_{4}{ }^{+}-\mathrm{N}$ and soil mineral $\mathrm{N}\left(\mathrm{NO}_{3}-\mathrm{N}+\mathrm{NH}_{4}{ }^{+}-\mathrm{N}\right)$ concentrations in the $0-30 \mathrm{~cm}$ soil layer at V3, V6, V10 and at silking, as well as corn grain yield were evaluated in both growing seasons. In general, $\mathrm{NO}_{3}{ }^{-}-\mathrm{N}$ soil concentrations were sensitive to the applied $\mathrm{N}$ rates, mainly in $\mathrm{V} 6$ when the best relationship between $\mathrm{NO}_{3}-\mathrm{N}$ and corn grain yield was found. The critical level of soil $\mathrm{NO}_{3}-\mathrm{N}$ concentration, above which a response to $\mathrm{N}$ application is unlikely, was estimated at $20 \mathrm{mg} \mathrm{kg}^{-1}$ in the studied soil, at growth stage V6. The determination of soil $\mathrm{NH}_{4}{ }^{+} \mathrm{N}$ concentration, in addition to soil $\mathrm{NO}_{3}{ }^{-}-\mathrm{N}$ concentration, improved the prediction of $N$ availability. A better relationship of soil mineral $\mathrm{N}\left(\mathrm{NH}_{4}^{+}-\mathrm{N}+\mathrm{NO}_{3}^{-}-\mathrm{N}\right)$ than of $\mathrm{NO}_{3}^{-}-\mathrm{N}$ concentration with corn grain yield was observed. These results indicate that the development of kits for instant determination of soil mineral $\mathrm{N}$ concentration is necessary. $\mathrm{NO}_{3}{ }^{-}-\mathrm{N}$ and $\mathrm{NH}_{4}{ }^{+}-\mathrm{N}$ as complementary indicators of soil $N$ availability for corn appear promising and studies should be developed under different climate and soil conditions to adequate their use to the management of sidedressed Nin corn.
\end{abstract}

Index terms: Zea mays, $N$ efficiency use, soil nitrate tests, grain yield, plant growth stages.

\section{INTRODUÇÃO}

No mundo, a eficiência de uso do $\mathrm{N}$ em cereais é de apenas $33 \%$. Considerando os $67 \%$ de $\mathrm{N}$ que não são aproveitados, há uma perda estimada anual de 15,9 bilhões de dólares com fertilização nitrogenada (Raun \& Johnson, 1999), em adição aos impactos negativos ao ambiente (Schröder et al., 2000). A alta mobilidade do $\mathrm{NO}_{3}{ }^{-}$no solo justifica a preocupação em relação ao manejo da adubação nitrogenada em solos agrícolas (Vanotti \& Bundy, 1994), e o aumento da concentração de $\mathrm{NO}_{3}{ }^{-}$na água tem gerado grande discussão sobre os seus efeitos na saúde e no ambiente, estimulando pesquisas de caráter agroecológico no mundo inteiro, principalmente em países desenvolvidos da Europa e da América do Norte (Addiscot, 2000; Andraski et al., 2000).

No Brasil, as baixas doses de $\mathrm{N}$ aplicadas nas culturas justificam, ao menos em parte, a menor preocupação quanto aos possíveis efeitos da fertilização nitrogenada no ambiente. Entretanto, o aumento das doses de $\mathrm{N}$ em sistemas altamente produtivos e o uso de fertilizantes nitrogenados associados com espécies leguminosas de plantas de cobertura e, ou, com dejetos animais merecem atenção quanto ao seu impacto no ambiente (Ceretta et al., 2003; Port et al., 2003), sobretudo a contaminação da água pela lixiviação de $\mathrm{NO}_{3}{ }^{-}$(Randall \& Mulla, 2001). 
O teor de matéria orgânica do solo (MOS) tem sido utilizado como o principal indicador da disponibilidade de N para as culturas no Brasil. Recentemente, no Sul do País, especificamente nos Estados do Rio Grande do Sul e de Santa Catarina, a recomendação de adubação nitrogenada em milho apresentou avanços expressivos, passando a considerar também o efeito das pré-culturas na disponibilidade de $\mathrm{N}$ (Amado et al., 2002). Considerando que o sistema de recomendação deve estar em constante aperfeiçoamento, uma melhoria potencial seria a inclusão de características complementares de solo e de planta que permitissem o monitoramento da disponibilidade de $\mathrm{N}$ durante o ciclo do milho, visando maior precisão na recomendação das doses e maior flexibilidade do manejo do $\mathrm{N}$ nessa cultura (Argenta, 2001; Rambo et al., 2004).

Dentre as características de solo usadas como indicadoras da disponibilidade de $\mathrm{N}$ no solo, o teor de $\mathrm{N}$ mineral, especialmente o de $\mathrm{NO}_{3}^{-}$, tem-se destacado. $\mathrm{O}$ uso desta característica baseia-se na disponibilidade de testes rápidos para sua determinação (Roth et al., 1992; Sims et al., 1995), bem como no fato de que grande proporção do $\mathrm{N}$ mineral está na forma de $\mathrm{NO}_{3}{ }^{-}$ (Sims et al., 1995; Ma \& Dwyer, 1999). No entanto, deve-se considerar que o íon $\mathrm{NO}_{3}{ }^{-}$é repelido pelas cargas negativas dos colóides, tendendo a permanecer em solução e ser lixiviado pela água de percolação (Dynia \& Camargo, 1999). Dessa forma, sob certas condições como, por exemplo, com alta precipitação pluvial ou sob irrigação complementar a inclusão da determinação do íon $\mathrm{NH}_{4}{ }^{+}$pode ser uma boa opção (Rambo, 2005), por encontrar-se adsorvido às cargas negativas do solo.

Os testes que fornecem ou indicam a quantidade de $\mathrm{N}$ mineral presente, denominados testes de intensidade, foram detalhamente discutidos em Rambo et al. (2004) e podem ser divididos basicamente em teste de pré-semeadura (TPS), teste de préaplicação de N em cobertura (TPNC) e teste de póscolheita (TPCC). Dos testes supracitados, destaca-se o TPNC, que tem sido o mais estudado e difundido, principalmente na América do Norte. Neste teste é avaliada a quantidade de $\mathrm{NO}_{3}{ }^{-}$que se encontra no solo até a profundidade de $30 \mathrm{~cm}$, no estádio V6 (seis folhas expandidas) de desenvolvimento de milho, visando detectar a variação da disponibilidade de $\mathrm{N}$ durante o ciclo da cultura e para predizer a dose de $\mathrm{N}$ a ser suplementada (Magdoff, 1991; Vanotti \& Bundy, 1994). Devem ser considerados vários aspectos que possibilitam que este teste possa predizer as necessidades de $\mathrm{N}$ da cultura do milho. Inicialmente, parte-se do princípio de que o $\mathrm{N}$ mineral no solo na época da amostragem é o resultado da integração de todos os fatores climáticos e de manejo da cultura, da adubação e do solo que influenciam a disponibilidade de $\mathrm{N}$ até a coleta. Considera-se também que grande parte do $\mathrm{N}$ do solo é mineralizada até pouco tempo antes da época de amostragem, que a maior parte do $\mathrm{N}$ disponível no solo deve se encontrar próximo à zona radicular no início do rápido crescimento da planta (V6), para assegurar nutrição adequada, e que o teor de $\mathrm{N}$ determinado pelo teste está correlacionado com o $\mathrm{N}$ total disponibilizado pelo solo ao longo da ontogenia da planta (Magdoff, 1991).

Poucos foram os estudos realizados no Brasil, sobretudo na região Sul, visando ao uso do teor de N mineral, principalmente o de $\mathrm{NO}_{3}{ }^{-}$, como indicador do teor de $\mathrm{N}$ no solo para a cultura do milho. Além disso, não se tem conhecimento de trabalhos realizados no Brasil que tenham utilizado essa abordagem no sistema plantio direto. Nesse sentido, este trabalho teve por objetivos: (a) avaliar o potencial de uso do teor de $\mathrm{N}-\mathrm{NO}_{3}{ }^{-}$no solo como indicador complementar da disponibilidade de $\mathrm{N}$ no solo em milho, (b) determinar o nível crítico de $\mathrm{N}_{-} \mathrm{NO}_{3}{ }^{-}$e o melhor estádio de desenvolvimento da cultura para sua avaliação no solo e (c) verificar se o uso do teor de $\mathrm{N}_{-} \mathrm{NH}_{4}{ }^{+}$no solo, em adição ao teor de $\mathrm{N}-\mathrm{NO}_{3}{ }^{-}$, aumenta a acurácia da predição da disponibilidade de N no solo para a cultura do milho.

\section{MATERIAL E MÉTODOS}

A pesquisa foi baseada em experimento realizado em campo, nos anos agrícolas 2002/03 e 2003/04, na Estação Experimental Agronômica (altitude de $46 \mathrm{~m}$ ) da Universidade Federal do Rio Grande do Sul (EEAUFRGS), região ecoclimática da Depressão Central, Eldorado do Sul, RS. O solo da área experimental é um Argissolo Vermelho (Embrapa, 1999). Como o Departamento de Plantas de Lavoura realiza rotação de áreas para evitar problemas relacionados ao monocultivo, o experimento foi realizado nos dois anos agrícolas em áreas distintas. No ano de 2002/03, o solo apresentou as seguintes características: argila: $290 \mathrm{~g} \mathrm{~kg}^{-1} ; \mathrm{pH}$ (água): 5,4; índice SMP: 5,9; P Mehlich-1: 9,4 $\mathrm{mg} \mathrm{dm}^{-3}$; K: $105 \mathrm{mg} \mathrm{L}^{-1}$; MOS: $25 \mathrm{~g} \mathrm{~kg}^{-1}$ e CTC: $7,6 \mathrm{cmol}_{\mathrm{c}} \mathrm{dm}^{-3}$. No ano de 2003/04, os resultados foram: argila: $380 \mathrm{~g} \mathrm{~kg}^{-1} ; \mathrm{pH}$ (água): 5,3; índice SMP: 6,2; P Mehlich-1: 5,6 mg dm${ }^{-3}$; $\mathrm{K}: 161 \mathrm{mg} \mathrm{dm}^{-3}$; MO: $25 \mathrm{~g} \mathrm{~kg}^{-1}$ e CTC: $8,1 \mathrm{cmol}_{\mathrm{c}} \mathrm{dm}^{-3}$, sendo todas as análises realizadas segundo procedimentos descritos em Tedesco et al. (1995).

Os tratamentos experimentais constaram da utilização de cinco doses de $\mathrm{N}$ na forma de uréia $(0,50$, 100,200 e $300 \mathrm{~kg} \mathrm{ha}^{-1}$ ), aplicadas parte na semeadura $(20 \%)$ e o restante no estádio V3 (três folhas expandidas, segundo Ritchie et al., 1993). O experimento foi disposto em blocos casualizados, com quatro repetições. O híbrido simples Pioneer 32R21, de ciclo superprecoce, foi semeado no primeiro ano em 15/10/ 2002 e, no segundo, em 27/10/2003, no espaçamento entre linhas de $0,7 \mathrm{~m}$ e na densidade de 65.000 sementes ha $^{-1}$, sobre os resíduos de aveia-preta (3,4 t ha ${ }^{-1}$ de matéria seca em $2002 / 03$ e $5,2 \mathrm{t} \mathrm{ha}^{-1}$ de matéria seca em 2003/04), em plantio direto. Em ambos os anos agrícolas a adubação com $\mathrm{P}$ e K foi 
realizada mecanicamente em linha, por ocasião da semeadura, tendo sido aplicados $80 \mathrm{~kg} \mathrm{ha}^{-1} \mathrm{de}_{2} \mathrm{O}_{5} \mathrm{e}$ $60 \mathrm{~kg} \mathrm{ha}^{-1}$ de $\mathrm{K}_{2} \mathrm{O}$ no primeiro ano e $105 \mathrm{~kg} \mathrm{ha}^{-1} \mathrm{de}$ $\mathrm{P}_{2} \mathrm{O}_{5}$ e $150 \mathrm{~kg} \mathrm{ha}^{-1}$ de $\mathrm{K}_{2} \mathrm{O}$ no segundo ano. Nos dois anos, o experimento foi irrigado por aspersão, quando o potencial de água no solo foi inferior a $-0,04 \mathrm{MPa}$. Foi realizado controle químico de plantas daninhas (estádio V3) e pragas (estádios V3 e V6), para que não interferissem no crescimento e na expressão do efeito das doses de $\mathrm{N}$ aplicadas no rendimento de grãos de milho.

As determinações realizadas nos dois anos agrícolas foram teores de $\mathrm{N}-\mathrm{NO}_{3}{ }_{3}$, de N- $\mathrm{NH}_{4}{ }^{+}$e de $\mathrm{N}$ mineral $\left(\mathrm{N}_{-} \mathrm{NO}_{3}{ }^{-}+\mathrm{N}-\mathrm{NH}_{4}{ }^{+}\right)$no solo e rendimento de grãos de milho. No primeiro ano, as características de solo foram avaliadas em quatro estádios de desenvolvimento do milho (V3 - três folhas expandidas, V6 - seis folhas expandidas, V10 - 10 folhas expandidas e espigamento), segundo escala proposta por Ritchie et al. (1993). No segundo ano, em função dos resultados obtidos no primeiro ano (Quadros 1 e 2), essas características foram avaliadas somente nos estádios V3 e V6.

Os teores de $\mathrm{N}_{-} \mathrm{NO}_{3}{ }^{-}$e de $\mathrm{N}-\mathrm{NH}_{4}{ }^{+}$no solo foram determinados em amostra composta, formada pela mistura de três subamostras de solo na camada de 0-30 cm de profundidade, por parcela (quatro linhas de $7 \mathrm{~m}$ ), coletadas nas entrelinhas do milho com trado calador. Nos estádios V3 e V6, nos dois anos agrícolas, as amostras de solo foram colocadas imediatamente após a coleta em béquer com $150 \mathrm{~mL}$ de KCl $1 \mathrm{~mol} \mathrm{~L}^{-1}$. Nos estádios V10 e espigamento, as amostras foram colocadas em sacos plásticos em caixa de isopor com gelo, sendo levadas ao laboratório e, em seguida, colocadas em béquer com $150 \mathrm{~mL}$ de $\mathrm{KCl} 1 \mathrm{~mol} \mathrm{~L}^{-1}$. Depois disso, em ambos os casos, as amostras foram agitadas por 30 min e deixadas decantar por mais $30 \mathrm{~min}$. Posteriormente, foi coletado o sobrenadante e determinados os teores de $\mathrm{N}_{-} \mathrm{NO}_{3}{ }^{-}$e $\mathrm{N}-\mathrm{NH}_{4}{ }^{+}$no solo, seguindo os procedimentos descritos por Tedesco et al (1995). O teor de $\mathrm{N}$ mineral foi obtido pela soma dos teores de $\mathrm{N}-\mathrm{NO}_{3}{ }^{-}$e de $\mathrm{N}-\mathrm{NH}_{4}{ }^{+}$no solo. O rendimento de grãos foi avaliado a partir da colheita de uma área útil de $8,4 \mathrm{~m}^{2}$ (duas linhas centrais de $6 \mathrm{~m}$ ) da parcela, sendo expresso numa umidade de $130 \mathrm{~g} \mathrm{~kg}^{-1}$.

Os indicadores de solo e o rendimento de grãos do milho foram submetidos à análise de variância pelo teste $\mathrm{F}$ a $5 \%$, bem como às análises de correlação e de regressão. Além disso, foi aplicada a análise gráfica proposta por Cate \& Nelson (1987), modificada por Fox et al. (2001), para determinação do nível crítico de $\mathrm{N}^{-\mathrm{NO}_{3}}{ }_{3}$ no solo no estádio V6. A dose de $\mathrm{N}$ a ser aplicada para cada unidade de $\mathrm{N}-\mathrm{NO}_{3}{ }^{-}$menor que a do nível crítico foi determinada de acordo com o procedimento descrito por Feibo et al. (1998).

Quadro 1. Teores médios de nitrato $\left(\mathrm{N}-\mathrm{NO}_{3}^{-}\right)$, amônio $\left(\mathrm{N}-\mathrm{NH}_{4}^{+}\right)$e de $\mathrm{N}$ mineral $\left(\mathrm{N}-\mathrm{NO}_{3}{ }^{-}+\mathrm{N}_{-} \mathrm{NH}_{4}^{+}\right)$no solo em diferentes estádios de desenvolvimento (M) e rendimento do milho, respectivos coeficientes de variação (CV) e significância do efeito das doses de $\mathrm{N}$ aplicadas (N), em dois anos agrícolas

\begin{tabular}{|c|c|c|c|c|c|c|c|c|c|c|c|c|}
\hline \multirow{3}{*}{ Característica avaliada } & \multicolumn{12}{|c|}{ Estádio de desenvolvimento(1) } \\
\hline & \multicolumn{3}{|c|}{ V3 } & \multicolumn{3}{|c|}{ V6 } & \multicolumn{3}{|c|}{ V10 } & \multicolumn{3}{|c|}{ Espigamento } \\
\hline & M & CV & $\mathbf{N}^{(2)}$ & M & CV & $\mathbf{N}$ & $\mathbf{M}$ & CV & $\mathbf{N}$ & $\mathbf{M}$ & $\mathrm{CV}$ & $\mathbf{N}$ \\
\hline & \multicolumn{3}{|c|}{$\%$} & \multicolumn{3}{|c|}{$\%$} & \multicolumn{3}{|c|}{$\%$} & \multicolumn{3}{|c|}{$\%$} \\
\hline & \multicolumn{12}{|c|}{ Ano agrícola 2002/03 } \\
\hline $\mathrm{N}^{-\mathrm{NO}_{3}}{ }^{-}$no solo $\left(\mathrm{mg} \mathrm{kg}^{-1}\right)$ & 8,15 & 11,5 & $* *$ & 17,94 & 11,4 & $* *$ & 20,00 & 28,3 & $\mathrm{~ns}$ & 20,50 & 10,5 & $\mathrm{~ns}$ \\
\hline $\mathrm{N}-\mathrm{NH}_{4}{ }^{+}$no solo $\left(\mathrm{mg} \mathrm{kg}^{-1}\right)$ & 11,54 & 22,9 & $\mathrm{~ns}$ & 21,63 & 10,8 & $* *$ & 12,71 & 37,4 & $\mathrm{~ns}$ & 13,80 & 25,6 & $\mathrm{~ns}$ \\
\hline $\mathrm{N}$ mineral no solo $\left(\mathrm{mg} \mathrm{kg}^{-1}\right)$ & 19,59 & 15,6 & $* *$ & 39,57 & 8,2 & $* *$ & 32,70 & 20,8 & * & 34,30 & 10,4 & * \\
\hline \multirow[t]{2}{*}{ Rendimento de grãos (t ha-1) } & 8,08 & 10,7 & $* *$ & & & & & & & & & \\
\hline & \multicolumn{12}{|c|}{ Ano agrícola 2003/04 } \\
\hline $\mathrm{N}^{-\mathrm{NO}_{3}}{ }^{-}$no solo $\left(\mathrm{mg} \mathrm{kg}^{-1}\right)$ & 8,65 & 19,9 & $\mathrm{~ns}$ & 12,75 & 16,3 & $* *$ & - & - & - & - & - & - \\
\hline $\mathrm{N}^{-\mathrm{NH}_{4}}{ }^{+}$no solo $\left(\mathrm{mg} \mathrm{kg}^{-1}\right)$ & 8,21 & 27,8 & $\mathrm{~ns}$ & 14,22 & 38,7 & * & - & - & - & - & - & - \\
\hline $\mathrm{N}$ mineral no solo $\left(\mathrm{mg} \mathrm{kg}^{-1}\right)$ & 16,87 & 19,5 & $\mathrm{~ns}$ & 26,96 & 26,9 & * & - & - & - & - & - & - \\
\hline Rendimento de grãos (t ha-1) & 7,39 & 15,8 & * & & & & & & & & & \\
\hline
\end{tabular}

\footnotetext{
(1) Segundo escala proposta por Ritchie et al. (1993). ${ }^{(2)}$ Fonte de variação: doses de N.

* $\mathrm{e}^{* *}$ : significativos a 5 e $1 \%$ pelo teste $\mathrm{F}$, respectivamente. ns: não-significativo a $5 \%$.
} 
Quadro 2. Coeficientes de correlação entre o rendimento de grãos do milho e os teores de nitrato $\left(\mathrm{N}^{-\mathrm{NO}_{3}}{ }^{-}\right)$, de amônio $\left(\mathrm{N}_{-} \mathrm{NH}_{4}{ }^{+}\right)$e de $\mathrm{N}$ mineral $\left(\mathrm{N}_{-} \mathrm{NO}_{3}{ }^{-}+\mathrm{N}_{-} \mathrm{NH}_{4}{ }^{+}\right)$no solo, por estádio de desenvolvimento, em dois anos agrícolas

\begin{tabular}{|c|c|c|c|c|}
\hline \multirow{2}{*}{$\begin{array}{c}\text { Estádio de } \\
\text { desenvolvimento }^{(1)}\end{array}$} & \multirow{2}{*}{ Ano agrícola } & \multicolumn{3}{|c|}{ Característica de solo $\left(\mathrm{mg} \mathrm{kg}^{-1}\right)$} \\
\hline & & Teor de $\mathrm{N}^{-\mathrm{NO}_{3}}{ }^{-}$ & Teor de N-NH${ }_{4}^{+}$ & Teor de $\mathrm{N}$ mineral \\
\hline & & \multicolumn{3}{|c|}{ Coeficiente de correlação (r) } \\
\hline \multirow[t]{2}{*}{ V3 } & $2002 / 03$ & $0,47^{*}$ & $0,32 \mathrm{~ns}$ & $0,41 \mathrm{~ns}$ \\
\hline & $2003 / 04$ & $0,42 \mathrm{~ns}$ & $0,42 \mathrm{~ns}$ & $0,47 *$ \\
\hline \multirow[t]{2}{*}{ V6 } & $2002 / 03$ & $0,57 * *$ & $0,70 * *$ & $0,69 * *$ \\
\hline & $2003 / 04$ & $0,71 * *$ & $0,70 * *$ & $0,74^{* *}$ \\
\hline \multirow[t]{2}{*}{ V10 } & $2002 / 03$ & $-0,29 \mathrm{~ns}$ & $-0,22 \mathrm{~ns}$ & $-0,33 \mathrm{~ns}$ \\
\hline & $2003 / 04$ & - & - & - \\
\hline \multirow[t]{2}{*}{ Espigamento } & $2002 / 03$ & $-0,12 \mathrm{~ns}$ & $0,08 \mathrm{~ns}$ & $0,0008 \mathrm{~ns}$ \\
\hline & $2003 / 04$ & - & - & - \\
\hline
\end{tabular}

${ }^{(1)}$ Segundo escala proposta por Ritchie et al. (1993). ${ }^{* *}$ e *: Significativos a 1 e $5 \%$ e ns: não-significativo a $5 \%$.

\section{RESULTADOS E DISCUSSÃO}

O rendimento de grãos do milho foi afetado pelas doses de $\mathrm{N}$ aplicadas nos dois anos de cultivo, apresentando média de 8,0 t ha-1 em 2002/03 e de 7,4 $\mathrm{t} \mathrm{ha}^{-1} \mathrm{em}$ 2003/04 (Quadro 1), as quais podem ser consideradas altas se comparadas com o rendimento médio obtido pelos produtores no RS. Os teores de N$\mathrm{NO}_{3}{ }^{-}$, de $\mathrm{N}-\mathrm{NH}_{4}{ }^{+}$e de $\mathrm{N}$ mineral $\left(\mathrm{N}-\mathrm{NO}_{3}{ }^{-}+\mathrm{N}^{-} \mathrm{NH}_{4}{ }^{+}\right)$ no solo foram responsivos às doses de $\mathrm{N}$ aplicadas, sendo a variação nos seus teores dependente do ano e do estádio de desenvolvimento em que foram avaliados. No primeiro ano, foram afetados significativamente o teor de $\mathrm{N}^{-\mathrm{NO}_{3}}{ }^{-}$no solo nos estádios $\mathrm{V} 3 \mathrm{e} \mathrm{V6}$, o teor de $\mathrm{N}-\mathrm{NH}_{4}{ }^{+}$no estádio V6 e o teor de $\mathrm{N}$ mineral nos quatro estádios avaliados. No segundo ano, em razão de os resultados obtidos no primeiro ano não terem mostrado boas relações dos indicadores de solo avaliados nos estádios V10 e espigamento com o rendimento de grãos, as avaliações restringiram-se aos estádios V3 e V6. Neste ano, todos os três indicadores de solo foram sensíveis em relação às doses de $\mathrm{N}$ aplicadas somente no estádio V6.

A fim de verificar a relação entre os indicadores de solo e o rendimento de grãos de milho, foram realizadas as análises de correlação e de regressão simples (Quadros 2 e 3). Verificou-se que os teores de $\mathrm{N}_{-} \mathrm{NO}_{3}{ }^{-}$, de $\mathrm{N}-\mathrm{NH}_{4}{ }^{+}$e de $\mathrm{N}$ mineral no solo apresentaram maiores correlações com rendimento de grãos nos estádios V3 e V6, com destaque para este último estádio (Quadro 2). Por outro lado, esses indicadores, quando avaliados nos estádios V10 e espigamento, não mostraram relação com o rendimento de grãos. Em razão disso, as análises de regressão dessas características com rendimento de grãos foram restringidas para os estádios V3 e V6 (Quadro 3), tendo sido verificada resposta quadrática do rendimento de grãos em relação aos teores de $\mathrm{N}^{-\mathrm{NO}_{3}}$, $\mathrm{N}-\mathrm{NH}_{4}{ }^{+}$e $\mathrm{N}$ mineral avaliados no estádio $\mathrm{V} 6$, bem como que as relações obtidas neste estádio foram bem superiores às do estádio V3 nos dois anos de estudo. Esses resultados evidenciam que a melhor época para determinação dos teores de N-NO${ }_{3}^{-}$, de N-NH${ }_{4}^{+}$e de $\mathrm{N}$ mineral, para predição da disponibilidade de $\mathrm{N}$ no solo, foi no estádio V6.

O uso do teor de $\mathrm{N}-\mathrm{NH}_{4}{ }^{+}$conjuntamente com o de $\mathrm{N}-\mathrm{NO}_{3}{ }^{-}$, formando o $\mathrm{N}$ mineral, de maneira geral, apresentou maior relação com rendimento de grãos que o teor de $\mathrm{N}_{-} \mathrm{NO}_{3}{ }^{-}$(Quadros 2 e 3). Isso indica que haveria maior acurácia na predição da disponibilidade de $\mathrm{N}$ no solo usando o teor de $\mathrm{N}$ mineral $\left(\mathrm{N}-\mathrm{NO}_{3}{ }^{-}+\mathrm{N}\right.$ $\mathrm{NH}_{4}{ }^{+}$) que somente o teor de $\mathrm{N}-\mathrm{NO}_{3}{ }^{-}$. Esses resultados diferem dos obtidos por Rozas et al. (2000) em trabalho desenvolvido na Argentina, no qual os autores observaram que a inclusão do teor de $\mathrm{N}_{-} \mathrm{NH}_{4}{ }^{+}$não aumentou significativamente a confiabilidade dos resultados. No entanto, em condições de intensa precipitação pluvial e em solos que receberam esterco animal ou fonte de $\mathrm{N}$ amoniacal, a avaliação do $\mathrm{N}-\mathrm{NH}_{4}{ }^{+}$também pode ser útil (Binford et al., 1992; Schmitt \& Randall, 1994).

Apesar de os resultados demonstrarem que se justificaria a determinação do teor de $\mathrm{N}_{-} \mathrm{NH}_{4}{ }^{+}$ juntamente ao de $\mathrm{N}-\mathrm{NO}_{3}{ }^{-}$para predição da disponibilidade de $\mathrm{N}$ no solo, a exeqüibilidade disso atualmente é baixa, pois não são disponíveis kits para avaliação de $\mathrm{N}$ mineral no solo. Com base nisso,

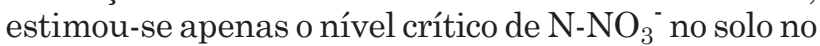
estádio V6, bem como a dose de $\mathrm{N}$ a ser aplicada para cada unidade de teor de $\mathrm{N}-\mathrm{NO}_{3}{ }^{-}$abaixo do nível crítico. 
O nível crítico de $\mathrm{N}^{-\mathrm{NO}_{3}}{ }^{-}$no solo foi determinado graficamente (Figura 1), a partir da relação entre o teor deste elemento no solo no estádio V6 e o rendimento de grãos relativo do milho, segundo os procedimentos estabelecidos por Cate \& Nelson (1987) e modificados por Fox et al. (2001). Assim, considerando como valor crítico horizontal o rendimento relativo de 0,93 e definindo-se o valor crítico vertical de modo a minimizar os erros ou outliers, estimou-se o valor crítico de $20 \mathrm{mg} \mathrm{kg}^{-1}$, a partir do qual a resposta à aplicação de N é pouco provável. De acordo com a análise gráfica, os dados que se encontram no quadrante superior esquerdo superestimaram a necessidade de $\mathrm{N}$, ou seja, indicaram que havia necessidade de $\mathrm{N}$ quando, na verdade, havia $\mathrm{N}$ suficiente para atingir o rendimento máximo. Já os dados que se encontram no quadrante inferior direito subestimaram a necessidade de N, ou seja, indicaram que não havia necessidade de $\mathrm{N}$ quando, de fato, o $\mathrm{N}$ era insuficiente para atingir o rendimento máximo.
O nível crítico de $20 \mathrm{mg} \mathrm{kg}^{-1}$ de $\mathrm{N}-\mathrm{NO}_{3}{ }^{-}$no solo encontra-se dentro da faixa de 15 a $30 \mathrm{mg} \mathrm{kg}^{-1}$ encontrada na literatura, cujo valor varia com a profundidade de amostragem, entre outros fatores (Rambo et al., 2004). Normalmente, menores valores são obtidos quando profundidades maiores de solo são amostradas, ocorrendo o contrário quando se amostram camadas menores (Rambo et al., 2004).

Com o objetivo de verificar a possibilidade de estabelecer doses de $\mathrm{N}$ em cobertura a partir dos teores de $\mathrm{N}$ mineral no solo, foram estabelecidas relações entre doses de $\mathrm{N}$ aplicadas e teor de $\mathrm{N}-\mathrm{NO}_{3}{ }^{-}$no solo, no estádio V6 do milho, nos dois anos agrícolas. Como o coeficiente angular foi o mesmo nos dois anos agrícolas, também foi estabelecida essa relação utilizando os dados obtidos nos dois anos conjuntamente (Quadro 4).

Empregando a equação entre doses de $\mathrm{N}$ aplicadas e teor de $\mathrm{N}-\mathrm{NO}_{3}{ }^{-}$no solo e nível crítico obtidos, seguindo o método usado por Feibo et al. (1998),

Quadro 3. Equações de regressão e coeficientes de determinação $\left(R^{2}\right)$ entre rendimento de grãos do milho e os teores $\left(\mathrm{mg} \mathrm{kg}^{-1}\right)$ de nitrato $\left(\mathrm{N}_{-} \mathrm{NO}_{3}^{-}\right)$, de amônio $\left(\mathrm{N}-\mathrm{NH}_{4}^{+}\right)$e de $\mathrm{N}$ mineral $\left(\mathrm{N}-\mathrm{NO}_{3}^{-}+\mathrm{N}_{-} \mathrm{NH}_{4}^{+}\right)$, em dois anos agrícolas

\begin{tabular}{|c|c|c|c|c|c|}
\hline \multirow{3}{*}{ Característica de solo } & \multirow{3}{*}{ Ano agrícola } & \multicolumn{4}{|c|}{ Estádio de desenvolvimento(1) $^{(1)}$} \\
\hline & & \multicolumn{2}{|l|}{ V3 } & \multicolumn{2}{|l|}{ V6 } \\
\hline & & Equação de regressão & $\mathbf{R}^{2}$ & Equação de regressão & $\mathbf{R}^{2}$ \\
\hline \multirow[t]{2}{*}{ Teor de $\mathrm{N}_{-} \mathrm{NO}_{3}^{-}$} & $2002 / 03$ & $\hat{\mathrm{Y}}=2542+699,74 \mathrm{x}$ & $0,22^{*}$ & $\hat{\mathrm{Y}}=-27699+3713,60 \mathrm{x}-93,59 \mathrm{x}^{2}$ & $0,40^{* *}$ \\
\hline & $2003 / 04$ & $\hat{\mathrm{Y}}=2465+568,98 \mathrm{x}$ & $0,14 \mathrm{~ns}$ & $\hat{\mathrm{Y}}=5145+1383,62 \mathrm{x}-29,44 \mathrm{x}^{2}$ & $0,51^{* *}$ \\
\hline \multirow[t]{2}{*}{ Teor de N-NH ${ }_{4}^{+}$} & $2002 / 03$ & $\hat{Y}=4979+268,62 x$ & $0,10 \mathrm{~ns}$ & $\hat{Y}=-6513+807,29 x-5,99 x^{2}$ & $0,49 * *$ \\
\hline & $2003 / 04$ & $\hat{\mathrm{Y}}=3629+457,64 \mathrm{x}$ & $0,16 \mathrm{~ns}$ & $\hat{\mathrm{Y}}=43,57+741,95 \mathrm{x}-13,00 \mathrm{x}^{2}$ & $0,53^{* *}$ \\
\hline \multirow[t]{2}{*}{ Teor de $\mathrm{N}$ mineral } & $2002 / 03$ & $\hat{\mathrm{Y}}=3353+240,06 \mathrm{x}$ & $0,17 \mathrm{~ns}$ & $\hat{\mathrm{Y}}=-4427+304,67 \mathrm{x}+0,28 \mathrm{x}^{2}$ & $0,48^{* *}$ \\
\hline & $2003 / 04$ & $\hat{Y}=2045+316,78 x$ & $0,19 \mathrm{~ns}$ & $\hat{\mathrm{Y}}=-1640+456,38 \mathrm{x}-4,01 \mathrm{x}^{2}$ & $0,56^{* *}$ \\
\hline
\end{tabular}

(1) Segundo escala proposta por Ritchie et al. (1993). Número de amostras por estádio de desenvolvimento $=20$.

** e *: Significativos a 1 e $5 \%$ e ns: não-significativo a $5 \%$.

Quadro 4. Equações de regressão e coeficientes de determinação $\left(\mathrm{R}^{2}\right)$ entre os teores de nitrato $\left(\mathrm{N}^{-N} \mathrm{NO}_{3}^{-}\right)$no solo (y, $\left.\mathrm{mg} \mathrm{kg}^{-1}\right)$ e níveis de $\mathrm{N}$ aplicados $\left(\mathrm{x}, \mathrm{kg} \mathrm{ha}^{-1}\right)$ no estádio $\mathrm{V} 6$ do milho, em dois anos agrícolas, e na análise conjunta dos anos

\begin{tabular}{cccc}
\hline Ano agrícola & Equação de regressão & $\mathbf{R}^{2}$ & $\mathbf{N}^{(1)}$ \\
\hline $2002 / 03$ & $\hat{\mathrm{Y}}=15,95+0,02 \mathrm{x}$ & $0,35^{* *}$ & 20 \\
$2003 / 04$ & $\hat{\mathrm{Y}}=9,82+0,02 \mathrm{x}$ & $0,54^{* *}$ & 20 \\
Conjunta & $\hat{\mathrm{Y}}=12,89+0,02 \mathrm{x}$ & $0,26^{* *}$ & 40 \\
\hline
\end{tabular}

(1) Numero de amostras. ** Significativo a $1 \%$. 
calculou-se a dose de $\mathrm{N}$ a ser aplicada para cada unidade de $\mathrm{N}^{-\mathrm{NO}_{3}}{ }^{-}$no solo abaixo do nível crítico. Inicialmente, foi calculada a dose de $\mathrm{N}$ para atingir o rendimento máximo de grãos, utilizando a equação conjunta (Quadro 4) e o nível crítico de $\mathrm{N}^{-\mathrm{NO}_{3}}{ }^{-}$no solo igual a $20 \mathrm{mg} \mathrm{kg}^{-1}$ (Figura 1), obtendo-se a dose de $365 \mathrm{~kg} \mathrm{ha}^{-1}$ de N. Posteriormente, fez-se esse mesmo cálculo usando o valor de $19 \mathrm{mg} \mathrm{kg}^{-1}$ de $\mathrm{N}$ $\mathrm{NO}_{3}{ }^{-}$no solo (uma unidade menor que a do nível crítico, obtendo-se o valor de $315 \mathrm{~kg} \mathrm{ha}^{-1}$ de N). Então, por diferença, pressupõe-se que, de acordo com esse método, seria necessária a aplicação de $50 \mathrm{~kg} \mathrm{ha}^{-1}$ para cada unidade de teor de $\mathrm{N}^{-} \mathrm{NO}_{3}{ }^{-}$no solo, medido no estádio V6, menor do que o do nível crítico, não havendo necessidade de aplicação de $\mathrm{N}$ quando o valor obtido for superior ao do nível crítico. No entanto, salientase que há necessidade de validação deste método em outros ambientes para sua utilização em campo, principalmente no que se refere à dose obtida.

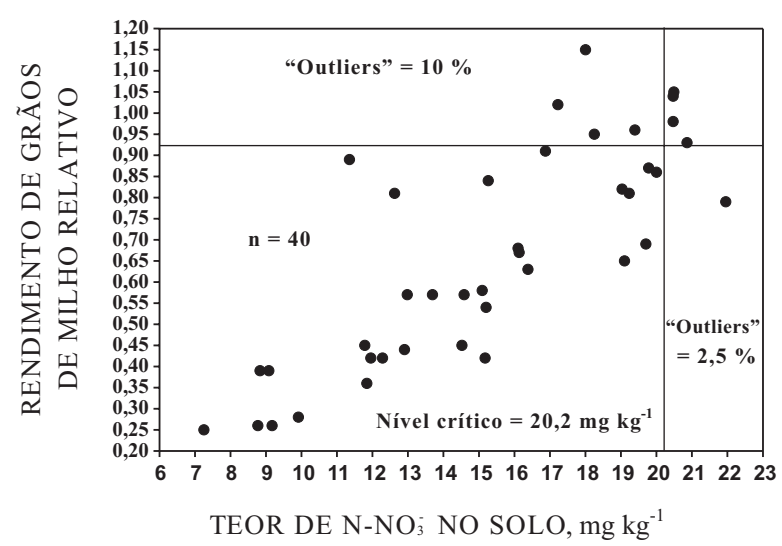

Figura 1. Determinação gráfica do nível crítico de nitrato $\left({\mathrm{N}-\mathrm{NO}_{3}}^{-}\right)$no solo, a partir da relação entre o teor de $\mathrm{N}-\mathrm{NO}_{3}^{-}$no solo e o rendimento relativo de grãos do milho.

$\mathrm{O}$ uso do TPNC como índice quantitativo da necessidade de adubação nitrogenada em cobertura em milho tem sido observado com sucesso nos Estados da Pensilvânia, Iowa e Vermont, nos Estados Unidos (Bundy \& Meisinger, 1994; Andraski \& Bundy, 2002). Já, em alguns casos, o seu uso como índice quantitativo tem sido comprometido, principalmente em razão da variabilidade na relação entre o teste e o rendimento relativo (Fox et al., 1989; Heckman et al., 1996). Devido a esses diferentes resultados, Bundy \& Meisinger (1994) recomendam que a adoção do TPNC sob o aspecto quantitativo deve ser avaliada em nível regional, não havendo perspectiva de que o uso deste teste de forma isolada seja eficiente para determinação da dose de $\mathrm{N}$ a ser aplicada, necessitando, portanto, de integração com outras características de solo e, ou, de planta.

\section{CONCLUSÕES}

1. O teor de $\mathrm{N}-\mathrm{NO}_{3}{ }^{-}$no solo tem potencial para ser utilizado como indicador complementar da disponibilidade de $\mathrm{N}$ no solo em milho.

2. A melhor época para determinação do teor de $\mathrm{N}-\mathrm{NO}_{3}{ }^{-}$no solo como indicador do nível de $\mathrm{N}$ no solo é no estádio V6.

3. O nível crítico do teor de $\mathrm{N}^{-\mathrm{NO}_{3}}{ }^{-}$no solo foi de $20 \mathrm{mg} \mathrm{kg}^{-1}$ para o ambiente estudado.

4. A utilização do teor de $\mathrm{N}_{-} \mathrm{NH}_{4}{ }^{+}$, em adição ao teor de $\mathrm{N}_{-} \mathrm{NO}_{3}^{-}$, aumenta a acurácia da predição da disponibilidade de N no solo para manejo da adubação nitrogenada em milho nas condições ambientais avaliadas, evidenciando a necessidade de desenvolvimento de kits de determinação rápida do teor de $\mathrm{N}$ mineral no solo.

\section{LITERATURA CITADA}

ADDISCTOTT, T.M. Tillage, mineralization and leaching foreword. Soil Till. Res., 53:163-165, 2000.

AMADO, T.J.C.; MIELNICZUK, J. \& AITA, C. Recomendação de adubação nitrogenada para o milho no RS e SC adaptada ao uso de culturas de cobertura do solo, sob sistema de plantio direto. R. Bras. Ci. Solo, 26:241-248, 2002 .

ANDRASKI, T.W. \& BUNDY, L.G. Using the pre-side dress soil nitrate test and organic nitrogen crediting to improve corn nitrogen recomendations. Agron. J., 94:1411-1418, 2002 .

ANDRASKI, T.W.; BUNDY, L.G. \& BRYE, K.R. Crop management and corn nitrogen rate effects on nitrate leaching. J. Environ. Quality, 29:1095-1103, 2000.

ARGENTA, G. Monitoramento do nível de nitrogênio na planta como indicador da adubação nitrogenada em milho. Porto Alegre, Universidade Federal do Rio Grande do Sul, 2001. 112p (Tese de Doutorado)

BINFORD, G.D.; BLACKMER, A.M. \& CERRATO, M.E. Relationships between corn yields and soil nitrate in late spring. Agron. J., 84:53-59, 1992.

BUNDY, L.G. \& MEISINGER, J.J. Nitrogen availability indices. In: WEAVER, R.W., ed. Methods of soil analysis. Part 2. Microbiological and biochemical properties. Madison, Soil Science Society of America, 1994. p.951-984.

CATE, R.B. \& NELSON, L.A. A simple statistical procedure for partitioning soil test correlation data into two classes. Soil Sci. Soc. Am. Proc., 35:658-660, 1987.

CERETTA, C.A.; DURIGON, R.; BASSO, C.J.; BARCELLOS, L.A.R. \& VIEIRA, F.C.B. Características químicas de solo sob aplicação de esterco líquido de suínos em pastagem natural. Pesq. Agropec. Bras., 38:729-735, 2003.

DYNIA, J.F. \& CAMARGO, O.A. Retenção de nitrato num solo de carga variável, influenciada por adubação fosfatada e calagem. Pesq. Agropec. Bras., 34:141-144, 1999. 
EMPRESA BRASILEIRA DE PESQUISA AGROPECUÁRIA . EMBRAPA. Centro Nacional de Pesquisa de Solos. Sistema brasileiro de classificação de solos. Brasília, Embrapa Produção da Informação; Rio de Janeiro, Embrapa Solos, 1999. 412p.

FEIBO, W.; LIANGHUAN, W. \& FUHUA, X. Chlorophyll meter to predict nitrogen sidedress requirements for shortseason cotton (Gossypium hirsutum L.). Field Crop Res., 56:309-314, 1998.

FOX, R.H.; PIEKIELEC, W.P. \& MACNEAL, K.E. Comparison of late-season diagnostic tests for predicting nitrogen status of corn. Agron., J., 93:590-597, 2001.

FOX, R.H.; ROTH, G.W.; IVERSEN, K.V. \& PIEKIELEK, W.P. Soil and tissue tests compared for predicting soil nitrogen availability to corn. Agron. J., 81:971-974, 1989.

HECKMAN, J.R.; GOVINDASAMY, R.; PROSTAK, D.J.; CHAMBERLAIN, E.A.; HLUBIK, W.T.; MICKEL, R.C. \& PROSTKO, E.P. Corn response to side dress nitrogen in relation to soil nitrate concentration. Comm. Soil Sci. Plant Anal., 27:575-583, 1996.

MA, B.L. \& DWYER, L.M. Within plot variability in available soil mineral nitrogen in relation to leaf greenness and yield. Comm. Soil Sci. Plant Anal., 30:1919-1928, 1999.

MAGDOFF, F. Understanding the Magdoff presidress nitrate test for corn. J. Produc. Agric., 3:297-305, 1991.

PORT, O.; AITA, C. \& GIACOMINI, S.J. Perda de nitrogênio por volatilização de amônia com o uso de dejetos de suínos em plantio direto. Pesq. Agropec. Bras., 38:857-865, 2003.

RAMBO, L. Integração de características de planta, de dossel e de solo para maior eficiência da adubação nitrogenada em cobertura em milho. Porto Alegre, Universidade Federal do Rio Grande do Sul, 2005. 176p. (Tese de Doutorado)

RAMBO, L.; SILVA, P.R.F.; ARGENTA, G. \& BAYER, C. Testes de nitrato no solo como indicadores complementares no manejo da adubação nitrogenada em milho. Ci. Rural, 34:1279-1287, 2004.
RANDALL, G. \& MULLA, D.J. Nitrate nitrogen in surface waters as influenced by climatic conditions and agriculture practices. Agron. J., 30:337-344, 2001.

RAUN, W.R. \& JOHNSON, G.V. Improving nitrogen use efficiency for cereal production. Agron. J., 91:357-363, 1999.

RITCHIE, S.W.; HANWAY, J.J. \& BENSON, G.O. How a corn plant develops? Ames, Iowa State University of Science and Technology, 1993. (Cooperative Extension Service. Special Report, 48)

ROTH, G.W.; BEEGLE, D.B. \& BOHN, P.J. Field evaluation of a presidress soil nitrate test and quick test for corn in Pennsylvania. J. Produc. Agric., 5:476-481, 1992.

ROZAS, H.S.; ECHEVERRÍA, H.E.; STUDDERT, G.A. \& DOMÍNGUEZ, G. Evaluation of the presidress soil nitrogen test for no-tillage maize fertilized at planting. Agron. J., 92:1176-1183, 2000.

SCHMITT, M.A. \& RANDALL, G.W. Developing a soil nitrogen test for improved recommendations for corn. J. Produc. Agric., 7:328-334, 1994.

SCHRÖDER, J.J.; NEETESON, J.J.; OENEMA, O. \& STRUIK, P.C. Does the crop or the soil indicate how to save nitrogen in maize production? Reviewing the state of art. Field Crops Res., 66:151-164, 2000.

SIMS, J.T.; VASILAS, B.L.; GARTLEY, K.L.; MILIKEN, B. \& GREEN, V. Evaluation of soil and plant nitrogen tests for maize on manured soils of the Atlantic coastal plain. Agron. J., 87:213-222, 1995.

TEDESCO, M.J.; GIANELLO, C.; BISSANI, C.A.; VOLKWEISS, S.J. \& BOHNEN, H. Análises de solo, plantas e outros materiais. 2.ed. Porto Alegre, Universidade Federal do Rio Grande do Sul, 1995. 174p.

VANOTTI, M.B. \& BUNDY, L.G. An alternative rationale for corn nitrogen fertilizer recommendations. J. Produc. Agric., 7:243-249, 1994. 